
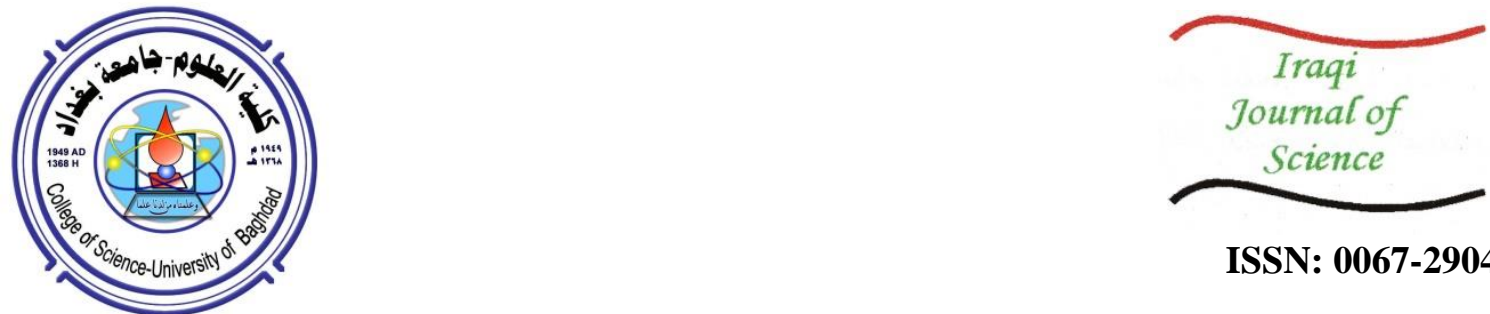

ISSN: 0067-2904

\title{
Efficient Modification of the Decomposition Method for Solving a System of PDEs
}

\author{
L. N. M. Tawfiq*, Z. H. Kareem \\ Department of Mathematics, College of Education for Pure Science Ibn Al-Haitham, University of Baghdad
}

Received: 8/9/2020 Accepted: 28/11/2020

\begin{abstract}
This paper presents an analysis solution for systems of partial differential equations using a new modification of the decomposition method to overcome the computational difficulties. Convergence of series solution was discussed with two illustrated examples, and the method showed a high-precision, being a fast approach to solve the non-linear system of PDEs with initial conditions. There is no need to convert the nonlinear terms into the linear ones due to the Adomian polynomials. The method does not require any discretization or assumption for a small parameter to be present in the problem. The steps of the suggested method are easily implemented, with high accuracy and rapid convergence to the exact solution, compared with other methods that can be used to solve systems of PDEs.
\end{abstract}

Keywords: System of PDEs, Decomposition Technique, Convergence Analysis.

\section{تعديل كفوء لطريقة التفكيك لحل منظومة معادلات تفاضلية جزئية}

\author{
زينب هادي كريم ، لمى ناجي عمحل توفيق \\ قسم الرياضيات، كلية التربية للعلوم الصرفة ابن الهيث؛، جامعله بغداد، بغدادن العراق لهاق
}

الخلاصة

يعرض البحث الحل التحليلي لهنظومة المعادلات التفاضلية الجزئية باستخدام تعديل جديد لطريقة

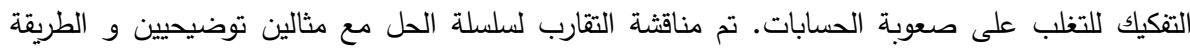

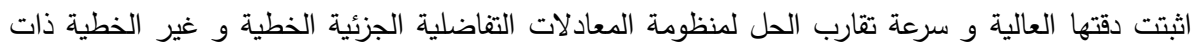

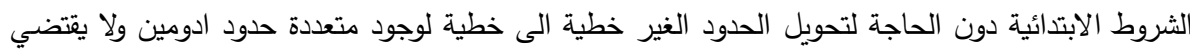

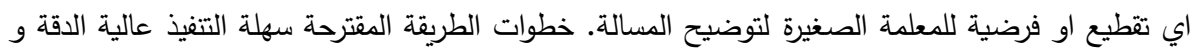

سريعة التقارب للحل المضبوط مقارنة مع الطرق الاخرى المستخدمة في حل منظومات المعادلات التفاضلية

الجزئية.

\section{Introduction}

The systems of partial differential equations (PDEs) have been used to describe many important models in real life, such as contamination, distribution of shallow water, heat, wave's contamination, and the chemical reaction - distribution model [1-4]. The general ideas and key characteristics of these systems are generally applicable [5]. In recent years, many authors have focused on solving the nonlinear systems of PDEs using various methods, such as Homotopy analysis method (HAM) [6], variational iteration method (VIM) [7], differential transform method (DTM) [8], Homotopy

*Email: luma.n.m@ihcoedu.uobaghdad.edu.iq 
perturbation method (HPM) [9-10], Adomain decomposition method (ADM) [11-13], coupled Laplace decomposition method [14], and semi analytic technique [15]. Recently, the decomposition method and its modifications have been used in a wider scope to solve different types of PDEs. In 2001, Wazwaz and Al-sayed [16] presented a modification of the ADM for non-linear operator, which replaced the process of dividing $f$ (non operator function) into two parts by an infinite series of components. Another modification is the restarted ADM [17]. In 2005, Wazwaz [18] found another modification to the ADM to overcome the difficulties that arise when the equation consists of singular points. This modification is useful for similar models with singularities. Luo [19] proposed another modification based on separating the ADM into two steps and, hence, it is termed the two steps ADM (TSAMD). The purpose behind the proposed scheme is to identify the exact solution more readily and eliminate some calculations. Herein we suggest a new modification for solving the non-linear systems of PDEs with initial conditions to overcome the computational difficulties.

\section{Description of the Suggested Modification}

The procedure of the suggested modification (MDM) to solve the non-linear system of PDEs is presented here. Firstly, we write the nonlinear system of PDEs as follows:

$L_{t} v+L_{X} v+N_{2}(u, v)=h_{2}(X, t)$

$$
L_{t} u+L_{X} u+N_{1}(u, v)=h_{1}(X, t)
$$

Subject to ICs:

$$
u(X, 0)=f(X) ; \quad v(X, 0)=g(X)
$$

where $X$ is a space variable that belongs in the $R, L:$ linear differential operator $\left(L_{t}=\frac{\partial}{\partial t}\right)$, $\left(L_{X}=\frac{\partial}{\partial x}\right), N_{1}$ and $N_{2}$ are nonlinear operators, and $h_{1}(X, t), h_{2}(X, t)$ are the nonhomogeneous part. By taking $L_{t}^{-1}=\int_{0}^{t}() d$.$t , to the system, we get$ $u(X, t)=f(X)+L_{t}^{-1}\left(h_{1}\right)-L_{t}^{-1} L_{X} u-L_{t}^{-1}\left[N_{1}(u, v)\right]$

$$
v(x, t)=g(X)+L_{t}^{-1}\left(h_{2}\right)-L_{t}^{-1} L_{X} v-L_{t}^{-1}\left[N_{2}(u, v)\right]
$$

The linear unknown functions $u(X, t)$ and $v(X, t)$ can be decomposed by infinite series of components, illustrated in equation (4), and $h_{1}, h_{2}$ should be decomposed infinite series illustrated in equation (5).

$$
\begin{aligned}
& u(X, t)=\sum_{m=0}^{\infty} a_{m}(X) t^{m}, v(X, t)=\sum_{m=0}^{\infty} b_{m}(X) t^{m} \\
& h_{1}=\sum_{m=0}^{\infty} r_{m}(X) t^{m} \text { and } h_{2}=\sum_{m=0}^{\infty} s_{m}(X) t^{m}
\end{aligned}
$$

$N_{1}(u, v), N_{2}(u, v)$ are nonlinear terms that can be represented by an infinite series of polynomials, as follows

$$
\begin{aligned}
& N_{1}(u, v)=\sum_{m=0}^{\infty} A_{m}(X) t^{m}=A_{0}+A_{1} t+A_{2} t^{2}+\cdots \\
& N_{2}(u, v)=\sum_{m=0}^{\infty} B_{m}(X) t^{m}=B_{0}+B_{1} t+B_{2} t^{2}+\cdots
\end{aligned}
$$

where $A_{m}$ and $B_{m}$ are Adomian polynomials

$A_{m}=\frac{1}{m !} \frac{d^{m}}{d \lambda^{m}}\left[N\left(\sum_{i=0}^{\infty} \lambda^{i} y_{i}\right)\right]_{\lambda=0}, \quad m=0,1,2, \ldots$.

By substituting (4) and (5) in the system (3), we get

$$
\begin{aligned}
& \sum_{m=0}^{\infty} a_{m}(X) t^{m}=f(x)+L_{t}^{-1}\left(\sum_{m=0}^{\infty} r_{m}(X) t^{m}\right)-L_{t}^{-1} L_{X}\left(\sum_{m=0}^{\infty} a_{m}(X) t^{m}\right)-L_{t}^{-1}\left(\sum_{m=0}^{\infty} A_{m}(X) t^{m}\right) \\
& \sum_{m=0}^{\infty} b_{m}(X) t^{m}=g(x)+L_{t}^{-1}\left(\sum_{m=0}^{\infty} s_{m}(X) t^{m}\right)-L_{t}^{-1} L_{x}\left(\sum_{m=0}^{\infty} b_{m}(X) t^{m}\right)-L_{t}^{-1}\left(\sum_{m=0}^{\infty} B_{m}(X) t^{m}\right)
\end{aligned}
$$

Now, we integrate the right side to get: 
$\sum_{m=0}^{\infty} a_{m}(X) t^{m}=f(x)+\left(\sum_{m=0}^{\infty} r_{m}(X) \frac{t^{m+1}}{m+1}\right)-L_{x}\left(\sum_{m=0}^{\infty} a_{m}(X) \frac{t^{m+1}}{m+1}\right)-\left(\sum_{m=0}^{\infty} A_{m}(X) t^{m} \frac{t^{m+1}}{m+1}\right)$

$\sum_{m=0}^{\infty} b_{m}(X) t^{m}=g(X)+\left(\sum_{m=0}^{\infty} s_{m}(X) \frac{t^{m+1}}{m+1}\right)-L_{X}\left(\sum_{m=0}^{\infty} b_{m}(X) \frac{t^{m+1}}{m+1}\right)-\left(\sum_{m=0}^{\infty} B_{m}(X) \frac{t^{m+1}}{m+1}\right)$

Let $m=m-1$ in the right side of the above system, we have

$\sum_{m=0}^{\infty} a_{m}(X) t^{m}=f(X)+\left(\sum_{m=1}^{\infty} r_{m-1}(X) \frac{t^{m}}{m}\right)-L_{x}\left(\sum_{m=1}^{\infty} a_{m-1}(X) \frac{t^{m}}{m}\right)-\left(\sum_{m=1}^{\infty} A_{m-1}(X) \frac{t^{m}}{m}\right)$

$\sum_{m=0}^{\infty} b_{m}(X) t^{m}=g(x)+\left(\sum_{m=1}^{\infty} s_{m-1}(X) \frac{t^{m}}{m}\right)-L_{x}\left(\sum_{m=1}^{\infty} b_{m-1}(X) \frac{t^{m}}{m}\right)-\left(\sum_{m=1}^{\infty} B_{m-1}(X) \frac{t^{m}}{m}\right)$

We have the following recursive relation:

$a_{0}(X)=f(X)$

$$
\text { Let } u_{0}=a_{0}, u_{1}=a_{1} t, u_{2}=a_{2} t^{2}, \ldots
$$

Let $w_{0}=b_{0}, w_{1}=b_{1} t, w_{2}=b_{2} t^{2}, \ldots$

After substituting in Adomian polynomials, we apply the relation (6):

In the same manner,

$$
a_{m}(X)=\frac{1}{m}\left[r_{m-1}(X)-\frac{\partial}{\partial x}\left(a_{m-1}(X)\right)-A_{m-1}(X)\right]
$$

$b_{0}(X)=g(X)$

$$
b_{m}(x)=\frac{1}{m}\left[s_{m-1}(X)-\frac{\partial}{\partial x}\left(b_{m-1}(X)\right)-B_{m-1}(X)\right]
$$

The coefficient $a_{m}(X), b_{m}(X)$ in eqs. (8) and (9) is substituted in eq. (4) to get the solution $u(X, t)$ and $v(X, t)$.

\section{Illustrative Problems}

In this section, the suggested modification (MDM) is used to solve the nonlinear system of PDEs.

\section{Problem 1}

Consider the following 2D nonlinear system of Burgers equation [20-21]:

$u_{t}+u u_{x}+w u_{y}=u_{x x}+u_{y y}$

$w_{t}+u w_{x}+w w_{y}=w_{x x}+w_{y y}$

subject to IC: $u(x, y, 0)=x+y, w(x, y, 0)=\mathrm{x}-\mathrm{y},(\mathrm{x}, \mathrm{y}, \mathrm{t}) \in R^{2} \times\left[0, \frac{1}{\sqrt{2}}\right)$.

\section{Solution}

By taking $L_{t}^{-1}=\int_{0}^{t}() d$.$t to the system, we obtain$

$$
\begin{aligned}
& u(x, y, t)=u(x, y, 0)+\frac{\partial^{2}}{\partial x^{2}} L_{t}^{-1}[u]+\frac{\partial^{2}}{\partial y^{2}} L_{t}^{-1}[u]-L_{t}^{-1}\left[u u_{x}\right]-L_{t}^{-1}\left[w u_{x}\right] \\
& w(x, y, t)=w(x, y, 0)+\frac{\partial^{2}}{\partial x^{2}} L_{t}^{-1}[w]+\frac{\partial^{2}}{\partial y^{2}} L_{t}^{-1}[w]-L_{t}^{-1}\left[u w_{x}\right]-L_{t}^{-1}\left[w u_{x}\right] \\
& u(x, y, t)=x+y+\frac{\partial^{2}}{\partial x^{2}} L_{t}^{-1}[u]+\frac{\partial^{2}}{\partial y^{2}} L_{t}^{-1}[u]-L_{t}^{-1}\left[u u_{x}\right]-L_{t}^{-1}\left[w u_{x}\right] \\
& w(x, y, t)=x-y+\frac{\partial^{2}}{\partial x^{2}} L_{t}^{-1}[w]+\frac{\partial^{2}}{\partial y^{2}} L_{t}^{-1}[w]-L_{t}^{-1}\left[u w_{x}\right]-L_{t}^{-1}\left[w u_{x}\right] \\
& \operatorname{let} u(x, t)=\sum_{m=0}^{\infty} a_{m}(x) t^{m}, w(x, t)=\sum_{m=0}^{\infty} b_{m}(x) t^{m} \\
& \sum_{m=0}^{\infty} a_{m}(x) t^{m}=x+y+\frac{\partial^{2}}{\partial x^{2}}\left(\sum_{m=0}^{\infty} a_{m}(x, y) \frac{t^{m+1}}{m+1}\right)+\frac{\partial^{2}}{\partial y^{2}}\left(\sum_{m=0}^{\infty} a_{m}(x, y) \frac{t^{m+1}}{m+1}\right) \\
& -\sum_{m=0}^{\infty} A_{m}(x, y) \frac{t^{m+1}}{m+1}-\sum_{m=0}^{\infty} B_{m}(x, y) \frac{t^{m+1}}{m+1}
\end{aligned}
$$




$$
\begin{aligned}
\sum_{m=0}^{\infty} b_{m}(x) t^{m}= & x-y+\frac{\partial^{2}}{\partial x^{2}}\left(\sum_{m=0}^{\infty} b_{m}(x, y) \frac{t^{m+1}}{m+1}\right)+\frac{\partial^{2}}{\partial y^{2}}\left(\sum_{m=0}^{\infty} b_{m}(x, y) \frac{t^{m+1}}{m+1}\right) \\
& -\sum_{m=0}^{\infty} C_{m}(x) \frac{t^{m+1}}{m+1}-\sum_{m=0}^{\infty} D_{m}(x, y) \frac{t^{m+1}}{m+1}
\end{aligned}
$$

where

$$
\begin{aligned}
& u(x, y, t)=\sum_{m=0}^{\infty} a_{m}(x, y) t^{m}, w(x, y, t)=\sum_{m=0}^{\infty} b_{m}(x, y) t^{m} \\
& u \frac{\partial u}{\partial x}=\sum_{\substack{m=0 \\
\infty}}^{\infty} A_{m}(x, y) t^{m}=A_{0}+A_{1} t+A_{2} t^{2}+\cdots
\end{aligned}
$$$$
w \frac{\partial u}{\partial y}=\sum_{m=0}^{\infty} B_{m}(x, y) t^{m}=B_{0}+B_{1} t+B_{2} t^{2}+\cdots
$$$$
u \frac{\partial w}{\partial x}=\sum_{m=0}^{\infty} C_{m}(x, y) t^{m}=C_{0}+C_{1} t+C_{2} t^{2}+\cdots
$$$$
w \frac{\partial w}{\partial y}=\sum_{m=0}^{\infty} D_{m}(x, y) t^{m}=D_{0}+D_{1} t+D_{2} t^{2}+\cdots
$$

Let $m=m-1$, then, on the right side of the above system, we have

$$
\begin{aligned}
\sum_{m=0}^{\infty} a_{m}(x) t^{m}= & x+y+\frac{\partial^{2}}{\partial x^{2}}\left(\sum_{m=1}^{\infty} a_{m-1}(x, y) \frac{t^{m}}{m}\right)+\frac{\partial^{2}}{\partial y^{2}}\left(\sum_{m=1}^{\infty} a_{m-1}(x, y) \frac{t^{m}}{m}\right) \\
& -\sum_{m=1}^{\infty} A_{m-1}(x, y) \frac{t^{m}}{m}-\sum_{m=1}^{\infty} B_{m-1}(x, y) \frac{t^{m}}{m} \\
\sum_{m=0}^{\infty} b_{m}(x) t^{m}= & x-y+\frac{\partial^{2}}{\partial x^{2}}\left(\sum_{m=1}^{\infty} b_{m-1}(x, y) \frac{t^{m}}{m}\right)+\frac{\partial^{2}}{\partial y^{2}}\left(\sum_{m=1}^{\infty} b_{m-1}(x, y) \frac{t^{m}}{m}\right) \\
& -\sum_{m=1}^{\infty} C_{m-1}(x) \frac{t^{m}}{m}-\sum_{m=1}^{\infty} D_{m-1}(x, y) \frac{t^{m}}{m}
\end{aligned}
$$

where $a_{0}=x+y$ and $b_{0}=x-y$

$a_{m}(x, y)=\frac{1}{m}\left[\frac{\partial^{2}}{\partial x^{2}}\left(a_{m-1}(x, y)\right)+\frac{\partial^{2}}{\partial y^{2}}\left(a_{m-1}(x, y)\right)-A_{m-1}(x, y)-B_{m-1}(x, y)\right]$

Also,

$b_{m}(x, y)=\frac{1}{m}\left[\frac{\partial^{2}}{\partial x^{2}}\left(b_{m-1}(x, y)\right)+\frac{\partial^{2}}{\partial y^{2}}\left(b_{m-1}(x, y)\right)-C_{m-1}(x, y)-D_{m-1}(x, y)\right]$
$a_{1}(x, y)=\frac{1}{1}\left[\frac{\partial^{2}}{\partial x^{2}}\left(a_{0}(x, y)\right)+\frac{\partial^{2}}{\partial y^{2}}\left(a_{0}(x, y)\right)-A_{0}(x, y)-B_{0}(x, y)\right]$

Let $u_{0}=a_{0}, u_{1}=a_{1} t, u_{2}=a_{2} t^{2}, \ldots$

$a_{0} a_{0 x}+\left(a_{1} a_{0 x}+a_{0} a_{1 x}\right) t+\left(a_{2} a_{0 x}+a_{1} a_{1 x}+a_{0} a_{2 x}\right) t^{2}+\cdots=A_{0}+A_{1} t+A_{2} t^{2}+\cdots$

Let $w_{0}=b_{0}, w_{1}=b_{1} t, w_{2}=b_{2} t^{2}, \ldots$

$b_{0} a_{0 y}+\left(b_{1} a_{0 y}+b_{0} a_{1 y}\right) t+\left(b_{2} a_{0 y}+b_{1} a_{1 y}+b_{0} a_{2 y}\right) t^{2}=B_{0}+B_{1} t+B_{2} t^{2}+\cdots$

$a_{0} b_{0 x}+\left(a_{1} b_{0 x}+a_{0} b_{1 x}\right) t+\left(a_{2} b_{0 x}+a_{1} b_{1 x}+a_{0} b_{2 x}\right) t^{2}+\cdots=C_{0}+C_{1} t+C_{2} t^{2}+\cdots$

$b_{0} b_{0 y}+\left(b_{1} b_{0 y}+b_{0} b_{1 y}\right) t+\left(b_{2} b_{0 y}+b_{1} b_{1 y}+b_{0} b_{2 y}\right) t^{2}+\cdots=D_{0}+D_{1} t+D_{2} t^{2}+\cdots$

$A_{0}(x, y)=x+y, B_{0}(x, y)=x-y$

$a_{1}(x, y)=[0+0-(x+y)-(x-y)]=[-x-y-x+y]=-2 x$

and so,

$b_{1}(x, y)=\frac{1}{1}\left[\frac{\partial^{2}}{\partial x^{2}}\left(b_{0}(x, y)\right)+\frac{\partial^{2}}{\partial y^{2}}\left(b_{0}(x, y)\right)-C_{0}(x, y)-D_{0}(x, y)\right]$ 


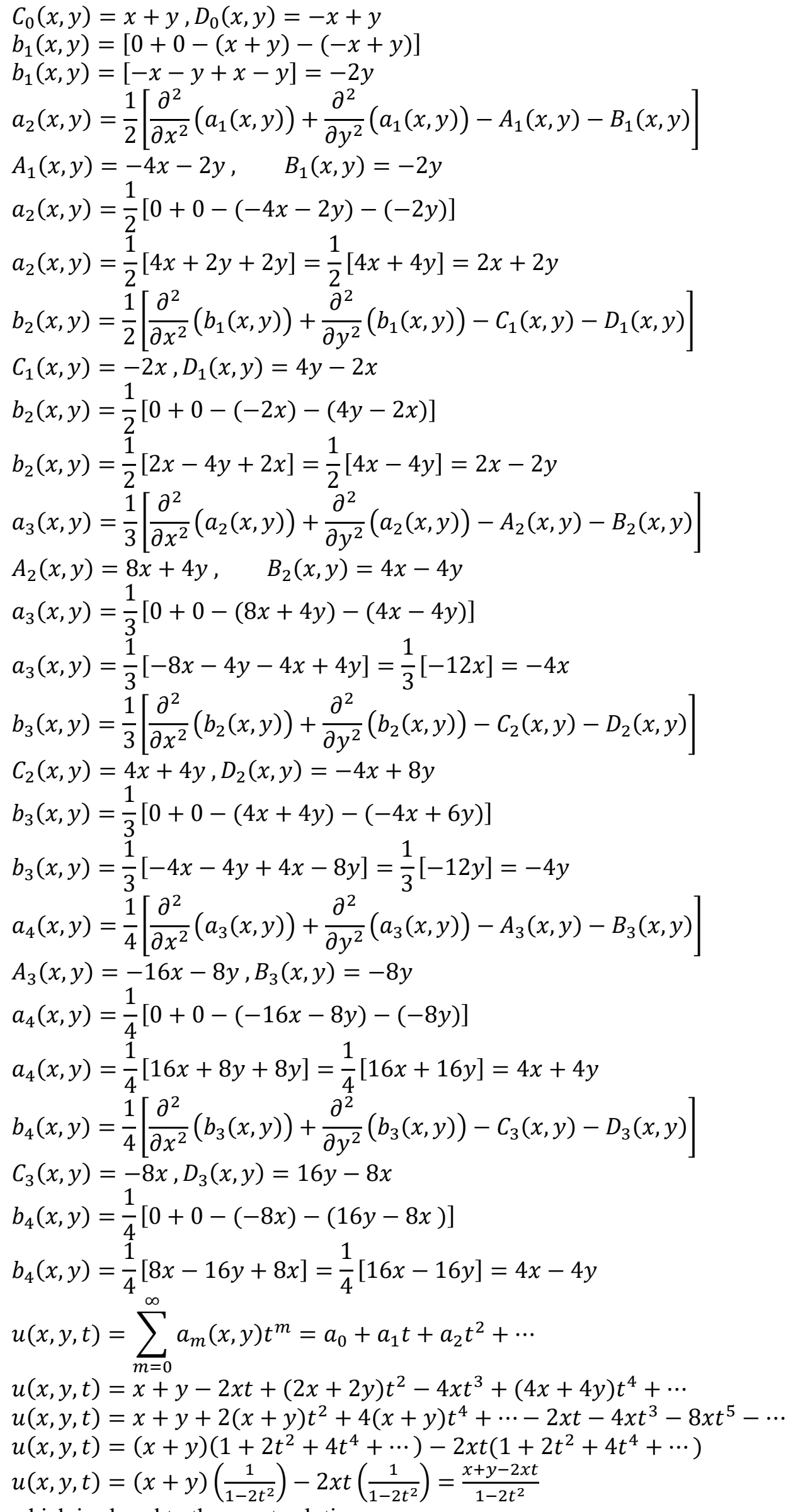

which is closed to the exact solution: 
$u(x, y, t)=\frac{x+y-2 x t}{1-2 t^{2}}$

and

$w(x, y, t)=\sum_{m=0}^{\infty} b_{m}(x, y) t^{m}=b_{0}+b_{1} t+b_{2} t^{2}+b_{3} t^{3}+b_{4} t^{4}+\cdots$

$w(x, y, t)=x-y-2 y t+(2 x-2 y) t^{2}-4 y t^{3}+(4 x-4 y) t^{4}+\cdots$

$w(x, y, t)=\left(x-y+2(x-y) t^{2}+4(x-y) t^{4}+8(x-y) t^{6}+\cdots\right)+\left(-2 y t-4 y t^{3}-8 y t^{5}-\cdots\right)$

$w(x, y, t)=(x-y)\left(1+2 t^{2}+4 t^{4}+8 t^{6}+\cdots\right)-2 y t\left(1+2 t^{2}+4 t^{4}+8 t^{6}+\cdots\right)$

$w(x, y, t)=(x-y)\left(\frac{1}{1-2 t^{2}}\right)-(2 y t)\left(\frac{1}{1-2 t^{2}}\right)=\frac{x-y-2 y t}{1-2 t^{2}}$

that is closed to the exact solution:

$w(x, y, t)=\frac{x-y-2 y t}{1-2 t^{2}}$

This problem was solved in [20-22] by using ADM and its modification. However, only a series solution, but not the exact solution, was obtained.

\section{Problem 2}

Consider a system of $3^{\text {rd }}$ order nonlinear PDE [23]

$u_{t}+v v_{x}=0$

$v_{t}+v_{x x x}+u v_{x}+u_{x} v=0$

subject to IC: $u(x, 0)=2 \operatorname{sech}^{2}(x), v(x, 0)=2 \operatorname{sech}(x)$

\section{Solution}

By taking $L_{t}^{-1}=\int_{0}^{t}() d$.$t to the system, we obtain$

$u(x, t)=u(x, 0)-L_{t}^{-1}\left[v v_{x}\right]$

$v(x, t)=v(x, 0)-\frac{\partial^{3}}{\partial x^{3}} L_{t}^{-1}[v]-L_{t}^{-1}\left[u v_{x}\right]-L_{t}^{-1}\left[u_{x} v\right]$

$u(x, t)=2 \operatorname{sech}^{2}(x)-L_{t}^{-1}\left[v v_{x}\right]$

$v(x, t)=2 \operatorname{sech}(x)-\frac{\partial^{3}}{\partial x^{3}} L_{t}^{-1}[v]-L_{t}^{-1}\left[u v_{x}\right]-L_{t}^{-1}\left[u_{x} v\right]$

Let $u(x, t)=\sum_{m=0}^{\infty} a_{m}(x) t^{m}, v(x, t)=\sum_{m=0}^{\infty} b_{m}(x) t^{m}$

$\sum_{m=0}^{\infty} a_{m}(x) t^{m}=2 \operatorname{sech}^{2}(x)-\left(\sum_{m=0}^{\infty} A_{m}(x) \frac{t^{m+1}}{m+1}\right)$

$\sum_{m=0}^{\infty} b_{m}(x) t^{m}=2 \operatorname{sech}(x)-\frac{\partial^{3}}{\partial x^{3}}\left(\sum_{m=0}^{\infty} b_{m}(x) \frac{t^{m+1}}{m+1}\right)-\sum_{m=0}^{\infty} B_{m}(x) \frac{t^{m+1}}{m+1}-\sum_{m=0}^{\infty} C_{m}(x) \frac{t^{m+1}}{m+1}$

where

$v v_{x}=\sum_{m=0}^{\infty} A_{m}(x) t^{m}=A_{0}+A_{1} t+A_{2} t^{2}+\cdots$

$u v_{x}=\sum_{m=0}^{\infty} B_{m}(x) t^{m}=B_{0}+B_{1} t+B_{2} t^{2}+\cdots$

$u_{x} v=\sum_{m=0}^{\infty} C_{m}(x) t^{m}=C_{0}+C_{1} t+C_{2} t^{2}+\cdots$

Let $m=m-1$ in the right side of the above system, then we have

$\sum_{m=0}^{\infty} a_{m}(x) t^{m}=2 \operatorname{sech}^{2}(x)-\left(\sum_{m=1}^{\infty} A_{m-1}(x) \frac{t^{m}}{m}\right)$

$\sum_{m=0}^{\infty} b_{m}(x) t^{m}=2 \operatorname{sech}(x)-\frac{\partial^{3}}{\partial x^{3}}\left(\sum_{m=1}^{\infty} b_{m-1}(x) \frac{t^{m}}{m}\right)-\sum_{m=1}^{\infty} B_{m-1}(x) \frac{t^{m}}{m}-\sum_{m=1}^{\infty} C_{m-1}(x) \frac{t^{m}}{m}$

$a_{0}(x)=2 \operatorname{sech}^{2}(x)$ 
$a_{m}(x)=\frac{1}{m}\left[-\left(A_{m-1}(x)\right)\right]$

Also,

$b_{0}(x)=2 \operatorname{sech}(x)$

$b_{m}(x)=\frac{1}{m}\left[-\frac{\partial^{3}}{\partial x^{3}}\left(b_{m-1}(x)\right)-B_{m-1}(x)-C_{m-1}(x)\right]$

Let $u_{0}=a_{0}, u_{1}=a_{1} t, u_{2}=a_{2} t^{2}, \ldots$

Let $v_{0}=b_{0}, v_{1}=b_{1} t, v_{2}=b_{2} t^{2}, \ldots$

$b_{0} b_{0 x}+\left(b_{1} b_{0 x}+b_{0} b_{1 x}\right) t+\left(b_{2} b_{0 x}+b_{1} b_{1 x}+b_{0} b_{2 x}\right) t^{2}+\cdots=A_{0}+A_{1} t+A_{2} t^{2}+\cdots$

$a_{0} b_{0 x}+\left(a_{1} b_{0 x}+a_{0} b_{1 x}\right) t+\left(a_{2} b_{0 x}+a_{1} b_{1 x}+a_{0} b_{2 x}\right) t^{2}+\cdots=B_{0}+B_{1} t+B_{2} t^{2}+\cdots$

$a_{0 x} b_{0}+\left(a_{1 x} b_{0}+a_{0 x} b_{1}\right) t+\left(a_{2 x} b_{0}+a_{1 x} b_{1}+a_{0 x} b_{2}\right) t^{2}+\cdots=C_{0}+C_{1} t+C_{2} t^{2}+\cdots$

$a_{1}(x)=\frac{1}{1}\left[-\left(A_{0}(x)\right)\right]$

Then

$a_{1}(x)=\frac{1}{1}\left[4 \tanh (x) \operatorname{sech}^{2}(x)\right]=4 \tanh (x) \operatorname{sech}^{2}(x)$

$b_{1}(x)=\frac{1}{1}\left[-\frac{\partial^{3}}{\partial x^{3}}\left(b_{0}(x)\right)-B_{0}(x)-C_{0}(x)\right]$

$b_{1}(x)=\left[-\frac{\partial^{3}}{\partial x^{3}}(2 \operatorname{sech}(x))+4 \tanh (x) \operatorname{sech}^{3}(x)+8 \tanh (x) \operatorname{sech}^{3}(x)\right]$

$b_{1}(x)=\left[-10 \tanh (x) \operatorname{sech}^{3}(x)+2 \tanh ^{3}(x) \operatorname{sech}(x)+4 \tanh (x) \operatorname{sech}^{3}(x)+8 \tanh (x) \operatorname{sech}^{3}(x)\right]$

$b_{1}(x)=\left[2 \tanh (x) \operatorname{sech}^{3}(x)+2 \tanh ^{3}(x) \operatorname{sech}(x)\right]$

$b_{1}(x)=\left[2 \tanh (x) \operatorname{sech}(x)\left(\operatorname{sech}^{2}(x)+\tanh ^{2}(x)\right)\right]$

$b_{1}(x)=2 \tanh (x) \operatorname{sech}(x)$

$a_{2}(x)=\frac{1}{2}\left[-\left(A_{1}(x)\right)\right]$

$a_{2}(x)=\frac{1}{2}\left[8 \tanh ^{2}(x) \operatorname{sech}^{2}(x)-4 \operatorname{sech}^{4}(x)\right]$

$a_{2}(x)=\frac{1}{2}\left[4 \operatorname{sech}^{2}(x)\left(2 \tanh ^{2}(x)-\operatorname{sech}^{2}(x)\right)\right]$

$a_{2}(x)=\frac{1}{2}\left[4 \operatorname{sech}^{2}(x)\left(\frac{\cosh (2 x)-1}{\cosh ^{2}(x)}-\frac{1}{\cosh ^{2}(x)}\right)\right]$

$a_{2}(x)=\frac{1}{2}\left[4 \operatorname{sech}^{2}(x)(-2+\cosh (2 x)) \operatorname{sech}^{2}(x)\right]$

$a_{2}(x)=\frac{1}{2}\left[4 \operatorname{sech}^{4}(x)(-2+\cosh (2 x))\right]$

$a_{2}(x)=-2 \operatorname{sech}^{4}(x)(2-\cosh (2 x))$

$b_{2}(x)=\frac{1}{2}\left[-\frac{\partial^{3}}{\partial x^{3}}\left(b_{1}(x)\right)-B_{1}(x)-C_{1}(x)\right]$

$b_{2}(x)=\frac{1}{2}\left[-\frac{\partial^{3}}{\partial x^{3}}(2 \tanh (x) \operatorname{sech}(x))+12 \tanh ^{2}(x) \operatorname{sech}^{3}(x)-4 \operatorname{sech}^{5}(x)\right.$

$\left.+24 \tanh ^{2}(x) \operatorname{sech}^{3}(x)-8 \operatorname{sech}^{5}(x)\right]$

$b_{2}(x)=\frac{1}{2}\left[10 \operatorname{sech}^{5}(x)-36 \tanh ^{2}(x) \operatorname{sech}^{3}(x)+2 \tanh ^{4}(x) \operatorname{sech}(x)+12 \tanh ^{2}(x) \operatorname{sech}^{3}(x)\right.$

$\left.-4 \operatorname{sech}^{5}(x)+24 \tanh ^{2}(x) \operatorname{sech}^{3}(x)-8 \operatorname{sech}^{5}(x)\right]$

$b_{2}(x)=\frac{1}{2}\left[-2 \operatorname{sech}^{5}(x)+2 \tanh ^{4}(x) \operatorname{sech}(x)\right]$

$b_{2}(x)=\frac{1}{2}\left[2 \operatorname{sech}(x)\left(-\operatorname{sech}^{4}(x)+\tanh ^{4}(x)\right)\right]$

$b_{2}(x)=\frac{1}{2}\left[2 \operatorname{sech}(x)\left(\tanh ^{4}(x)-\operatorname{sech}^{4}(x)\right)\right]$ 


$$
\begin{aligned}
& b_{2}(x)=\frac{1}{2}\left[2 \operatorname{sech}(x)\left(\tanh ^{2}(x)-\operatorname{sech}^{2}(x)\right)\right] \\
& b_{2}(x)=\frac{1}{2}\left[2 \operatorname{sech}(x)\left(\frac{\frac{1}{2}(\cosh (2 x)-1)}{\cosh ^{2}(x)}-\frac{1}{\cosh ^{2}(x)}\right)\right] \\
& b_{2}(x)=\frac{1}{2}\left[2 \operatorname{sech}^{3}(x)\left(\frac{1}{2} \cosh (2 x)-\frac{3}{2}\right)\right] \\
& b_{2}(x)=\frac{1}{2}\left[\operatorname{sech}^{3}(x)(\cosh (2 x)-3)\right] \\
& b_{2}(x)=\frac{1}{2}\left[\operatorname{sech}^{3}(x)(3-\cosh (2 x))\right] \\
& b_{2}(x)=-\frac{1}{2}(3-\cosh (2 x)) \operatorname{sech}^{3}(x)
\end{aligned}
$$

and so on

$$
\begin{aligned}
& u(x, t)=\sum_{m=0}^{\infty} a_{m}(x) t^{m}=a_{0}+a_{1} t+a_{2} t^{2}+\cdots \\
& u(x, t)=2 \operatorname{sech}^{2}(x)+\left(4 \operatorname{ctanh}(x) \operatorname{sech}^{2}(x)\right) t-\left(2 \operatorname{sech}^{4}(x)(2-\cosh (2 x))\right) t^{2}+\cdots
\end{aligned}
$$$$
v(x, t)=\sum_{m=0}^{\infty} b_{m}(x) t^{m}=b_{0}+b_{1} t+b_{2} t^{2}+\cdots
$$$$
v(x, t)=2 \operatorname{sech}(x)+(2 \tanh (x) \operatorname{sech}(x)) t-\frac{1}{2}(3-\cosh (2 x)) \operatorname{sech}^{3}(x) t^{2}+\cdots
$$

that is closed to the exact solution:

$$
u(x, t)=2 \operatorname{sech}^{2}(x-t), \quad v(x, t)=2 \operatorname{sech}(x-t)
$$

Problem 2 is solved in [24] by using ADM and its modification, but only the series solution, not the exact solution was obtained.

\section{Convergence Analysis of the Series Solution}

In this section, the convergence analysis of the series solution for the non-linear systems of PDEs is discussed. The sufficient requirement for convergence of the suggested modification is addressed. We show that the series solution for the systems of PDEs is converge to the exact solution.

\section{Definition 1 [20]}

A Banach space is a complete, normed, Vector space.

All norms on a Vector space of finite dimensions are equivalent. Every finite-dimensional standard space is a Banach space, over $\mathbf{R}$ or $\mathbf{C}$.

\section{Definition 2 [21]}

Let $X$ be a set and let $f: x \rightarrow x$ be a function that maps $x$ into itself. Such a function is often called an operator. A fixed point of $f$ is an element $x \in X$, for which $f(x)=x$.

\section{Definition 3 [25]}

Let $(X, d)$ be a metric space. A mapping $T: X \rightarrow X$ is a contraction mapping, or contraction, if there exists a constant $\mathrm{c}$, with $0 \leq \mathrm{c}<1$, such that

$d(T(x), T(y)) \leq c d(x, y), x, y \in X$

\section{Definition 4 [26]}

Let $(X, d)$ be a complete metric space. A mapping $T: X \rightarrow X$ is a nonlinear contraction mapping, or nonlinear contraction, if there exists a constant $\mathrm{c}$, with $0 \leq \mathrm{c}<1$, such that $d(T(x), T(y)) \leq c(d(x, y)) d(x, y), x, y \in X$.

Theorem 5 (Banach's fixed-point theorem)

A contractive function $T$ on a Banach space $S$ has a Unique fixed point $X^{*}$ in $R^{2}$ [27].

\section{Theorem 6 (Sufficient Condition for Convergence)}

If $\mathrm{X}$ and $\mathrm{Y}$ are Banach spaces and $N: X \rightarrow Y$ is a contractive nonlinear mapping, that is $\forall w, w^{*} \in X ;\left\|N(w)-N\left(w^{*}\right)\right\| \leq \gamma\left\|w-w^{*}\right\|, 0<\gamma<1$,

then, according to Banach's fixed-point theorem, $\mathrm{N}$ has a unique fixed-point $\mathrm{U}$, that is $N(u)=\mathrm{U}$. Assume that the sequence generated by the suggested method can be written as $w_{n}=N\left(w_{n-1}\right), w_{n-1}=\sum_{i=0}^{n-1} w_{i}, n=1,2,3, \ldots$ 
Suppose that $W_{0}=w_{0} \in B_{r}(w)$ where $B_{r}(w)=\left\{w^{*} \in X \mid\left\|w^{*}-w\right\|<r\right\}$, then we have i. $w_{n} \in B_{r}(w)$

ii. $\lim _{n \rightarrow \infty} W_{n}=w$

Proof

(i) By the inductive approach, for $n=1$, we have

$\left\|W_{1}-w\right\|=\left\|N\left(W_{0}\right)-N(w)\right\| \leq \gamma\left\|w_{0}-w\right\|$

Assume that $\left\|W_{n-1}-w\right\| \leq \gamma\left\|w_{n-1}-w\right\|$

$$
\begin{aligned}
& \leq \gamma^{2}\left\|w_{n-2}-w\right\| \\
& \leq \gamma^{3}\left\|w_{n-3}-w\right\|
\end{aligned}
$$$$
\leq \gamma^{n-1}\left\|w_{0}-w\right\|
$$

As in the induction hypothesis, then

$\left\|W_{n}-w\right\|=\left\|N\left(W_{n-1}\right)-N(w)\right\| \leq \gamma\left\|w_{n-1}-w\right\| \leq \gamma^{n}\left\|w_{0}-w\right\|$

Using (i), we have

$\left\|W_{n}-w\right\| \leq \gamma^{n}\left\|w_{0}-w\right\| \leq \gamma^{n} r<r \Rightarrow W_{n} \in B_{r}(w)$

Because of $\left\|W_{n}-w\right\| \leq \gamma^{n}\left\|w_{0}-w\right\|$ and

$\lim _{n \rightarrow \infty} \gamma^{n}=0, \lim _{n \rightarrow \infty}\left\|W_{n}-w\right\|=0$

that is

$\lim _{n \rightarrow \infty} W_{n}=w$

\section{Conclusions}

In this article, a new modification of the decomposition method is suggested to solve the nonlinear system. We obtained an exact analytical solution, where ADM or other modifications are used to solved the same examples but cannot achieve an exact analytical solution. Moreover, $u_{0}$ in ADM and its modification is $u_{0}=f(x)+t g(x)$, versus $u_{0}=a_{0}=f(x), a_{1}=g(x)$ in MDM, which is the main reason for simplifying the steps of solution. Moreover, in MDM, the nonlinear terms are easier to compute than in ADM or its modifications. The convergence concept of the decomposition series was thoroughly investigated to confirm the rapid convergence of the resulting series. Hence, this approach is very efficient, with easy implementation and rapid convergence to the exact solutions.

\section{References}

1. Kareem ZH, Tawfiq LNM. 2020. Recent Modification of Decomposition Method for Solving Nonlinear Partial Differential Equations. Journal of Advances in mathematics. 18: 154-161.

2. Kareem ZH, Tawfiq LNM. 2020. Solving Three-Dimensional Groundwater Recharge Based on Decomposition Method. Journal of Physics: Conference Series. 1530(012068):1-8.

3. Wazwaz A.M. 2002. Partial differential equations: Methods and applications, Balkema Publishers, The Netherlands;

4. Tawfiq LNM. Altaie H. 2020. Recent Modification of Homotopy Perturbation Method for Solving System of Third Order PDEs. Journal of Physics: Conference Series. 1530 (012073): 1-8.

5. Hussein, NA., and Tawfiq, LNM. 2021. New Approach for Solving (2+1)-Dimensional Differential Equation, Journal of Physics: Conference Series, 1818, 012182, 1-13.

6. Tawfiq, LNM., and Ibrahim Abed, AQ. 2021. Efficient Method for Solving Fourth Order PDEs, Journal of Physics: Conference Series, 1818, 012166, 1-10.

7. He J. H. and Wu X. H. 2007. Variational iteration method: New development and applications. Computers \& Mathematics with Applications. 54(7-8): 881-894.

8. Salih H., and Tawfiq LNM. 2020. Solution of Modified Equal Width Equation Using Quartic Trigonometric-Spline Method. Journal of Physics: Conference Series. 1664, 012033: 1-10.

9. Hussein, NA and Tawfiq, LNM. 2020. New Approach for Solving (1+1)-Dimensional Differential Equation. Journal of Physics: Conference Series. 1530, 012098, 1-11

10. Ghazi FF, Tawfiq LNM. 2020. New Approach for Solving Two Dimensional Spaces PDE. Journal of Physics: Conference Series. 1530 (012066): 1-10.

11. Tawfiq LNM. Ali M H. 2020. Efficient Design of Neural Networks for Solving Third Order Partial. Journal of Physics: Conference Series. 1530 (012067): 1-8.

12. Enadi M O, Tawfiq, LNM. 2019. New Technique for Solving Autonomous Equations. Ibn AlHaitham Journal for Pure and Applied Science. 32(2): 123-130. 
13. Enadi MO, Tawfiq LNM. 2019. New Approach for Solving Three Dimensional Space Partial Differential Equation. Baghdad Science Journal. 16(3): 786-792.

14. Jasem F. 2011. Application of laplace-adomian decomposition method on linear and nonlinear system of PDEs. Applied Mathematical Sciences. 5(27): 1307-1315.

15. Tawfiq L.N.M. and Hilal M.M. 2017. Solution of 2nd Order Nonlinear Three-Point Boundary Value Problems By Semi-Analytic Technique. IHJPAS. 27(3).

16. Wazwaz A.-M. and El-sayed, S. M. 2001. A New Modification of the Adomian Decomposition Method for Linear and Nonlinear Operators. Applied Mathematics and Computation. 122 (3): 393405.

17. Zwillinger D. 1997. Handbook of Differential Equations. 3rd edition Academic Press.

18. Wazwaz A.M. 2005. Adomian decomposition method for a reliable treatment of the EmdenFowler equation. Applied Mathematics and Computation. 161: 543-560.

19. Tawfiq, LNM. and Salih, OM. 2019. Design neural network based upon decomposition approach for solving reaction diffusion equation Journal of Physics: Conference Series 1234, 012104, 1-8.

20. Wazwaz A.-M. 2006. The modified decomposition method for analytic treatment of differential equations. Appl. Math. Comput. 173(1): 165-176.

21. Tawfiq, L.N.M, Al-Noor, N.H. and Al-Noor, T. H. 2019. Estimate the Rate of Contamination in Baghdad Soils By Using Numerical Method, Journal of Physics: Conference Series, 1294, 032020, 1-8.

22. Tawfiq LNM, Hassan MA. 2018. Estimate the Effect of Rainwaters in Contaminated Soil by Using Simulink Technique. In Journal of Physics: Conference Series. 1003 (012057):1-7.

23. Tawfiq LNM, Jabber AK. 2018. Steady State Radial Flow in Anisotropic and Homogenous in Confined Aquifers. Journal of Physics: Conference Series. 1003 (012056): 1-12.

24. Tawfiq LNM, Abood I N. 2018. Persons Camp Using Interpolation Method. Journal of Physics: Conference Series. 1003 (012055): 1-10.

25. Salih, H., Tawfiq, LNM., Yahya, ZRI and Zin, S M. 2018. Solving Modified Regularized Long Wave Equation Using Collocation Method Journal of Physics: Conference Series, 1003, 012062, 110.

26. Nuseira, I., Shatanawib, W., Abu-Irwaqa, I., Bataihah, A. 2017. Nonlinear contractions and fixed point theorems with modified $\omega$-distance mappings in complete quasi metric spaces. J. Nonlinear Sci. Appl., 10: 5342-5350.

27. Tawfiq, L.N.M, Al-Noor, N.H. and Al-Noor, T. H. 2019. Estimate the Rate of Contamination in Baghdad Soils By Using Numerical Method, Journal of Physics: Conference Series, 1294, 032020: $1-8$. 